\title{
Influence of nanoparticulated chitosan on the biomodification of eroded dentin: clinical and photographic longitudinal analysis of restorations
}

\author{
José Caetano de Souza ${ }^{1}$ - Antônio Cláudio Tedesco ${ }^{2} \cdot$ Luandra Aparecida Unten Takahashi $^{2}$.

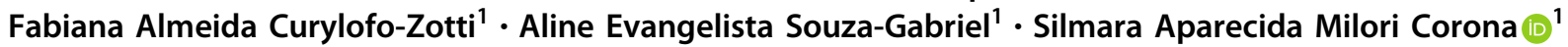

Received: 29 June 2020 / Accepted: 18 December 2020 / Published online: 20 January 2021

(c) The Author(s) 2021

\begin{abstract}
To evaluate the influence of the pre-treatment with $2.5 \%$ nanoparticulate chitosan (2.5\% NanoChi) solution on eroded dentin before the restorative dental treatment. The sample consisted of 22 patients (age between 33 and 52 years) with shallow or medium erosion lesions located in two homologous teeth. The teeth were randomly assigned according to dentin treatment: with $2.5 \%$ NanoChi and without with chitosan (control). The NanoChi were applied immediately after acid etching. The teeth were restored with Single Bond Universal (3 M) and Charisma resin (Kulzer). Analyzes were done using modified USPHS (retention, secondary caries, marginal adaptation, and sensitivity) and photographic (color, marginal pigmentation, and anatomical form) criteria at 7 days (baseline) and 1 year. Population demographics, Kaplan-Meier estimates and logrank test (Mantel-Cox) were calculated for 1 year $(\alpha=0.05)$. No significant difference was found in the survival rates between groups $(p>0.05)$ at 7 days and 1 year after treatment. After 7 days, $100 \%$ of the restorations were scored as Alpha on all criteria. After 1 year, 91\% of the NanoChi restorations were scored as Alpha and 9\% as Charlie for the retention, marginal adaptation, and anatomical form criteria, while $86 \%$ of the control restorations (without NanoChi) received the Alpha score and $14 \%$ received the Charlie. Secondary caries, sensitivity, color, and marginal pigmentation criteria were scored as Alpha in $100 \%$ of the restorations. The biomodification of eroded dentin with $2.5 \%$ NanoChi did not influence the survival of the restorations after 1 year. The application of 2.5\% NanoChi on eroded dentin did not increase failures of resin restorations after 1 year and it can be used as a pre-treatment solution.
\end{abstract}

\section{Introduction}

Dental erosion is the irreversible and progressive loss of tooth structure caused by non-bacterial chemical processes with different etiological factors [1] that allow it to be classified as intrinsic and extrinsic. Intrinsic erosion occurs

Silmara Aparecida Milori Corona

silmaracorona@forp.usp.br

1 Department of Restorative Dentistry, Ribeirão Preto School of Dentistry, University of São Paulo, Ribeirão Preto, São Paulo 14040-904, Brazil

2 Department of Chemistry, Center of Nanotechnology and Tissue Engineering, Photobiology and Photomedicine Research Group, Faculty of Philosophy, Sciences and Letters of Ribeirão Preto, University of São Paulo, Ribeirão Preto, São Paulo 14040-901, Brazil when teeth get into contact with gastric acid during recurrent vomiting or gastroesophageal reflux [2]. Extrinsic erosion is caused by exogenous acids from diet or drugs [3]. The prevalence of dental erosion has increased in industrialized countries [4], especially in overweight youth and children [5], due to the high intake of energy and isotonic drinks by athletes and citrus fruits [6].

The erosion process can affect both enamel and dentin. In enamel, the demineralization of the inorganic phase of the tooth exposes the prisms and reduces microhardness [7]. The dissolution of the peritubular portion and demineralization of intertubular dentin exposes the organic matrix, forming a rough and porous surface that increases the enzymatic degradation of collagen, therefore, impairing the adhesive interface in the restorative therapy $[8,9]$.

Dentin collagen degradation has generally been associated with poor clinical performance of composite resin restorations [10]. The adhesion between dental tissue and 
restoration is compromised in dentin exposed to mechanical or chemical challenges [11]. In eroded dentin, the high water content of collagen fibers hampers the adhesive infiltration and accelerates the degradation of the adhesive interface[12]. Previous studies suggest the use of natural solutions to modify the dental surface, such as chitosan, aiming to increase the mechanical resistance of collagen fibrils to degradation, providing support for the adhesive interface [13-15].

Chitosan, a hydrophilic biopolymer obtained by deacetylation of chitin $[16,17]$, is the most abundant polysaccharide in nature after cellulose [18], and has properties such as biocompatibility, bioadhesion, biodegradability, low human cell toxicity, and antimicrobial activity [17, 19], as well as chelating capacity [20], providing greater longevity in eroded dentin restorations [9]. Chitosan can increase the number of crosslinks between collagen fibers and neutralize metalloproteinases from dentin [16].

This study aimed to evaluate the influence of $2.5 \% \mathrm{w} / \mathrm{w}$ nanoparticulate chitosan (2.5\% NanoChi) solution on eroded dentin biomodification at baseline ( 7 days) and final (1 year) periods before the restorative treatment, by clinical and photographic examination using modified United States Public Health Service (USPHS) criteria.

\section{Materials and methods}

\subsection{Experimental design}

For the experiment, 22 patients with erosive noncarious lesions located on the palatal or lingual surface of two homologous teeth $(n=22)$ were selected according to the randomized block design. The two teeth of each patient were divided into two groups: restoration with $2.5 \%$ NanoChi and without NanoChi (control). Restorations were evaluated clinically and photographically using modified USPHS criteria at baseline ( 7 days) and final ( 1 year) after treatment.

\subsection{Sample size calculation and ethical aspects}

To determine the sample size, we used the power calculation function of the sample size calculation based on the protocol previously described in www.sealedenvelope.com, using the trial equivalent with the following parameters: $\alpha$ $=5 \%$, power $90 \%$, success percentage of control and experimental groups of $98 \%$ and equivalence limit of $15 \%$, reaching a sample size of 19 restorations per group.

The project was approved by the local Research Ethics Committee (79949317.1.0000.5419) and was registered at the Brazilian Registry of Clinical Trials (RBR-22md2n). Each patient signed the informed consent form.

\subsection{Preparation of NanoChi solution}

The preparation of $2.5 \%$ NanoChi was carried out at the Center for Nanotechnology and Tissue Engineering of the Ribeirão Preto School of Philosophy, Sciences and Letters (FFCLRP) from commercially available chitosan (SigmaAldrich, Saint Louis, MO, USA), low molecular weight (75-85\% deacetylation).

Chitosan nanoparticles reached an average size of less than $300 \mathrm{~nm}$. The polydispersity index varied between 0.311 and 0.422 , which indicates nanoparticles of different sizes. The zeta potential is around $+30 \mathrm{mV}$, considered strongly cationic. The presence of amino groups in the polymeric chain prevents the aggregation of the nanoparticles. Chitosan nanoparticles containing green tea reached an average size of less than $350 \mathrm{~nm}$. The polydispersity index is smaller than 0.45 , and the zeta potential is around $+40 \mathrm{mV}$. Few variations along the 80 -day period evaluated to indicate the long-term stability of the nanoformulation.

\subsection{Patients and teeth selection}

Thirty-one patients aged between 33 and 52 years of both sexes were examined at the Dental Clinic of the Ribeirão Preto School of Dentistry. Patients underwent prophylaxis with pumice paste (SS White, Rio de Janeiro, RJ, Brazil), water and rubber cup (Jon, São Paulo, SP, Brazil) on smooth surfaces and Robinson brush (Jon, São Paulo, SP, Brazil) on occlusal surfaces, using a micromotor handpiece (Gnatus, Ribeirão Preto, SP, Brazil). Waxed dental floss (Aperibé, RJ, Brazil) was used on the proximal surfaces. Clinical examination was performed under adequate lighting after prophylaxis.

The inclusion criterion was the presence of at least two shallow or medium erosion lesions located on the palatal or lingual surface of homologous vital teeth. Tooth vitality was tested with the thermal test with Endofrost (Roeko, Langenau, Germany). Of the examined patients, 22 were selected. The medical history and dental charts were completed, and patients received individualized instructions on diet and oral hygiene. Patients with temporomandibular dysfunction, bruxism, teeth with pain, spontaneous tenderness, fistula, or edema were excluded from the study. The patients who were not selected for the study but needed restorative treatment were referred to our school's dentistry clinic.

\subsection{Treatment of erosion lesions}

Patients' teeth were randomized into two groups using a computer spreadsheet and a random number generator available at the website http://randomnumbergenerator.intemodino. com/en. Prophylaxis was performed as previously described 
and initial photographs of the teeth were taken (buccal and palatal/lingual) (Canon EOS Rebel T2i 18.0 Megapixels, Cannon, Japan).

The color of the Charisma composite resin (Kulzer South America, Sao Paulo SP, Brazil) was then selected using the Vita 3D color scale (Wilcos do Brasil Industria e Comercio Ltda., Petrópolis, RJ, Brazil). Absolute isolation of the teeth was performed with a rubber dam (Madeitex, São Jose dos Campos, SP, Brazil) and clamps (Duflex, SS White, Rio de Janeiro, RJ, Brazil), according to the morphology of each tooth.

The enamel was acid-etched with $35 \%$ phosphoric acid for $30 \mathrm{~s}$, and dentin was not etched. After the cavity was washed with water for $1 \mathrm{~min}$, the excess water was removed with the suction cannula, and the surface dried with absorbent paper. In the experimental group, the $2.5 \%$ NanoChi was actively applied on the dentin with a single disposable microbrush (KGBrush, KG Sorensen, Cotia, SP, Brazil) for $1 \mathrm{~min}$, followed by washing with water and drying with absorbent paper.

The layer of the Single Bond Universal adhesive system (3 M ESPE, Sao Paulo, SP, Brazil) was actively applied with a disposable microbrush (KGBrush, KG Sorensen, Cotia, SP, Brazil) for $10 \mathrm{~s}$ and light-cured (Gnatus, Ribeirão Preto, SP, Brazil). Charisma composite resins (Kulzer South America, São Paulo SP, Brazil) were used through the incremental restoration technique with a resin spatula, and each increment was light-cured for $20 \mathrm{~s}$. Occlusal contact points were recorded with carbon paper (Angelus, Londrina, PR, Brazil) after tooth restoration. Premature contacts and occlusal interferences were removed using diamond burs (KG Sorensen, Cotia, SP, Brazil). Subsequently, diamond burs (KG Sorensen, Cotia, SP, Brazil) and polishing disks (TDV, Praxis, Santa Catarina, SC, Brazil) were used for finishing. The patients returned after 7 days for final polishing of the restoration with abrasive burs (Dentsply, Petrópolis, RJ, Brazil) and pumice paste (SS White, Rio de Janeiro, RJ, Brazil).

\subsection{Clinical and photographic analyzes of restorations}

Clinical and photographic analyzes of restorations were performed by three calibrated and experienced examiners long-term followed up evaluation at the 1-year interval, following the modified USPHS clinical (retention, secondary caries, marginal adaptation, and postoperative sensitivity) and photographic (restoration color, marginal pigmentation, and anatomical form) criteria [21]. The same dentist performed all restorations after training and calibration of the clinical protocol (Fig. 1). The clinical and photographic evaluations of the restorations (baseline and 1 year) were performed by three evaluators (dentists who did not participate in the restoration) after training and calibration.

The restorations were scored into Alpha-when the evaluated criterion had no problems, and the restoration was in perfect condition; Bravo-when the evaluated criterion had minor but clinically acceptable failures and Charliewhen the evaluated criterion had relevant failures and the restoration needed to be replaced (Table 1).

Intraoral photographs were taken at both time-points with the digital camera (Canon EOS Rebel T2i 18.0 Megapixels, Cannon, Japan) and a buccal photography mirror (Indusbello, Londrina, PR, Brazil), recording the buccal and palatal/lingual surfaces of the restored teeth, totaling two photos for each treatment. Photographic analyzes of restorations were performed by examiners viewing the images on a laptop screen, under the same environment and lighting.

\subsection{Data analysis}

Statistical analysis was performed using SPSS statistical software for Windows version 25.0 (SPSS Inc., Chicago, IL, USA). Survival analysis by the Kaplan-Meier method and log-rank test (Mantel-Cox) were performed with the scores obtained during the clinical and photographic analyzes at both time-points. Cohen's kappa coefficient was used to check the agreement level between operators.

\section{Results}

Forty-four restorations (100\% of the studied groups) were analyzed in 22 patients ( 7 men and 15 women) with a mean age of 40.5 years (range 33-52 years). The followup period was 1 year. Eight upper central incisors, ten upper lateral incisors, eight upper canines, six lower central incisors, six lower lateral incisors, and six lower canines were included in the study (Table 2). There was no statistically significant difference in the survival of restorations among men and women $(p=0.072)$, different age groups $(p=0.780)$, or position in the dental arch $(p=0.193)$.

No statistically significant difference $(p>0.05)$ was found between restorations with and without eroded dentin biomodification at baseline (7 days) and final (1 year) periods after treatment $(p=0.836)$. The evaluation of dental restorations obtaine an intra and inter examiner kappa of 1.0 (intra examiner kappa $A=1, B=1$ and $C=1$; inter examiner kappa $A \times B=1, A \times C=0.95$, and $C \times B=0.92$ ), with perfect agreement among examiners.

After 7 days of the procedure, $100 \%$ of the restorations were scored as Alpha in all clinical (retention, secondary 

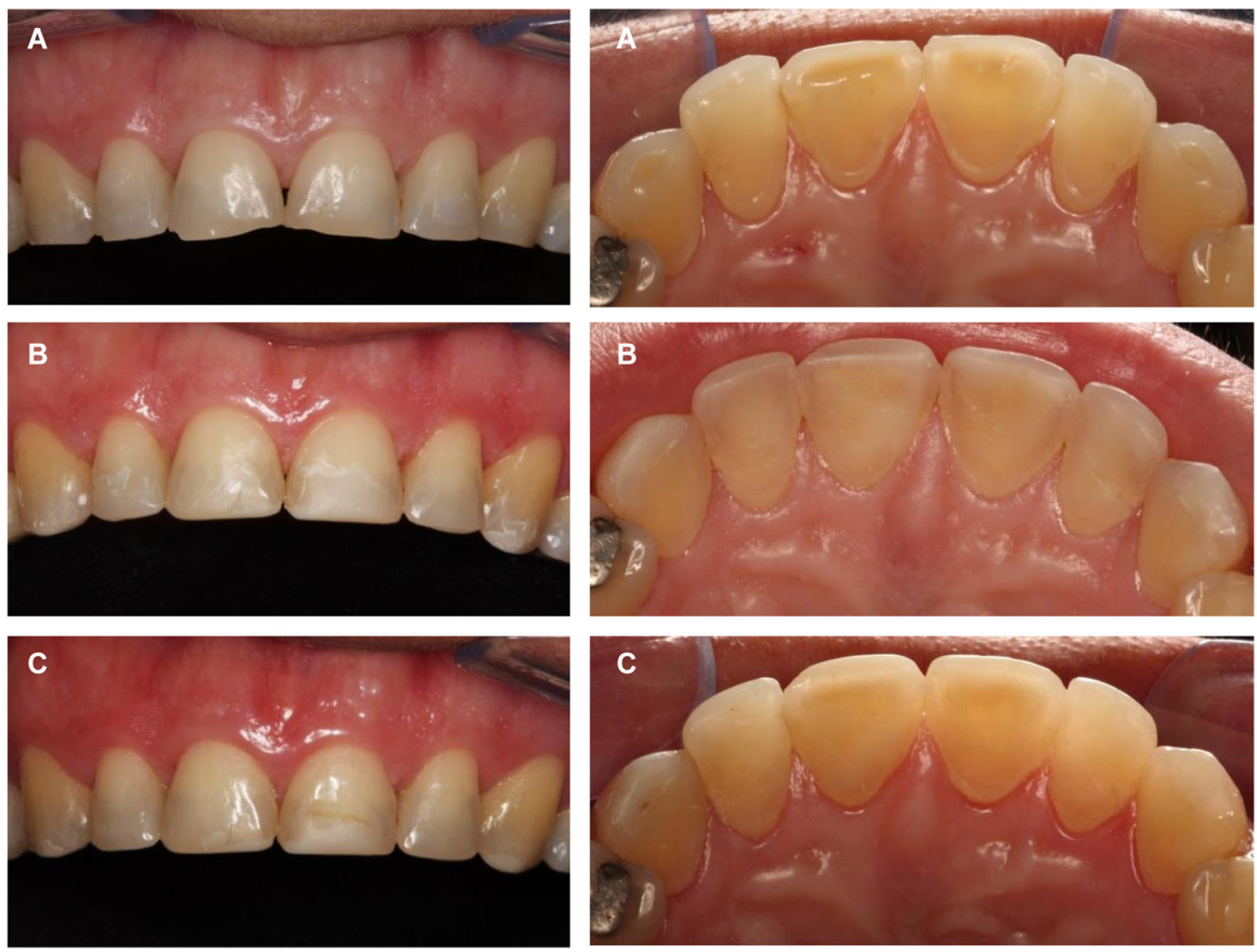

Fig. 1 Clinical aspects of erosion lesions and restorations. a Buccal and palatal erosion lesions-clinical appearance (b) 7 days and (c) 1 year after treatment. The right central incisor was from the control

group (without biomodification) and the left central incisor was from the test group (with $2.5 \%$ NanoChi)

Table 1 Modified USPHS criteria used for the clinical and photographic analysis of restorations

\begin{tabular}{|c|c|c|c|}
\hline Clinical criteria & Score & $\begin{array}{l}\text { Photographic } \\
\text { criteria }\end{array}$ & Score \\
\hline Retention & $\begin{array}{l}\text { A. No loss of restorative material } \\
\text { B. Partial loss of restorative material } \\
\text { C. Total loss of restorative material }\end{array}$ & Restoration color & $\begin{array}{l}\text { A. Corresponds to adjacent dental structure in terms of } \\
\text { color and translucency } \\
\text { B. Slight change in color, shade, or translucency } \\
\text { between restoration and adjacent tooth } \\
\text { C. Clear color change and translucency }\end{array}$ \\
\hline Secondary caries & $\begin{array}{l}\text { A. No recurrence of caries } \\
\text { B. With recurrence of superficial caries } \\
\text { C. With recurrence of deep caries }\end{array}$ & $\begin{array}{l}\text { Marginal } \\
\text { pigmentation }\end{array}$ & $\begin{array}{l}\text { A. No pigmentation along margin between restoration } \\
\text { and adjacent tooth } \\
\text { B. Slight pigmentation along the margin between the } \\
\text { restoration and adjacent tooth } \\
\text { C. Pigmentation present along restoration margin }\end{array}$ \\
\hline $\begin{array}{l}\text { Marginal } \\
\text { adaptation }\end{array}$ & $\begin{array}{l}\text { A. Perfectly adaptable with no visible margins } \\
\text { B. Visible but clinically acceptable margin } \\
\text { C. Marginal mismatch, clinical failure }\end{array}$ & Anatomic form & $\begin{array}{l}\text { A. Restoration in continuity with existing } \\
\text { anatomical form } \\
\text { B. Restoration in discontinuity with anatomical form }\end{array}$ \\
\hline $\begin{array}{l}\text { Postoperative } \\
\text { Sensitivity }\end{array}$ & $\begin{array}{l}\text { A. Missing stimulated sensitivity. } \\
\text { B. Present and localized stimulated sensitivity. } \\
\text { C. Present and diffuse stimulated sensitivity }\end{array}$ & & $\begin{array}{l}\text { of tooth } \\
\text { C. Loss of material by exposing dentin or } \\
\text { restoration base }\end{array}$ \\
\hline
\end{tabular}

$A$ Alpha, $B$ Bravo, $C$ Charlie scores

caries, marginal adaptation, and postoperative sensitivity) and photographic (restoration color, marginal pigmentation, and anatomical form) criteria.
After 1 year of the procedure, 91\% of the restorations in the experimental group were scored as Alpha and 9\% as Charlie for retention and marginal adaptation clinical 
Table 2 Profiles of the patients attended

\begin{tabular}{|c|c|c|c|c|c|c|c|c|}
\hline Patient & $\operatorname{Sex}(M / F)$ & Age & $\begin{array}{l}\text { Restored } \\
\text { tooth }(+)\end{array}$ & Bone base & Lesion depth & $\begin{array}{l}\text { Restored } \\
\text { tooth }(-)\end{array}$ & Bone base & Lesion depth \\
\hline 1 & M & 33 & 21 & Maxilla & Medium & 11 & Maxilla & Medium \\
\hline 2 & $\mathrm{~F}$ & 47 & 21 & Maxilla & Medium & 11 & Maxilla & Medium \\
\hline 3 & $\mathrm{~F}$ & 39 & 21 & Maxilla & Medium & 11 & Maxilla & Medium \\
\hline 4 & $\mathrm{~F}$ & 52 & 21 & Maxilla & Medium & 11 & Maxilla & Medium \\
\hline 5 & $\mathrm{~F}$ & 40 & 22 & Maxilla & Medium & 12 & Maxilla & Medium \\
\hline 6 & M & 34 & 22 & Maxilla & Medium & 12 & Maxilla & Medium \\
\hline 7 & $\mathrm{~F}$ & 51 & 22 & Maxilla & Medium & 12 & Maxilla & Medium \\
\hline 8 & M & 37 & 22 & Maxilla & Shallow & 12 & Maxilla & Shallow \\
\hline 9 & $\mathrm{~F}$ & 46 & 22 & Maxilla & Shallow & 12 & Maxilla & Shallow \\
\hline 10 & $\mathrm{~F}$ & 39 & 23 & Maxilla & Medium & 13 & Maxilla & Medium \\
\hline 11 & M & 38 & 23 & Maxilla & Shallow & 13 & Maxilla & Shallow \\
\hline 12 & $\mathrm{~F}$ & 52 & 23 & Maxilla & Shallow & 13 & Maxilla & Shallow \\
\hline 13 & $\mathrm{~F}$ & 47 & 23 & Maxilla & Shallow & 13 & Maxilla & Shallow \\
\hline 14 & $\mathrm{~F}$ & 40 & 31 & Mandible & Medium & 31 & Mandible & Medium \\
\hline 15 & F & 45 & 31 & Mandible & Shallow & 31 & Mandible & Shallow \\
\hline 16 & M & 38 & 41 & Mandible & Shallow & 41 & Mandible & Shallow \\
\hline 17 & M & 40 & 32 & Mandible & Medium & 42 & Mandible & Medium \\
\hline 18 & F & 46 & 32 & Mandible & Shallow & 42 & Mandible & Shallow \\
\hline 19 & F & 39 & 32 & Mandible & Shallow & 42 & Mandible & Shallow \\
\hline 20 & M & 41 & 33 & Mandible & Medium & 43 & Mandible & Medium \\
\hline 21 & F & 42 & 33 & Mandible & Shallow & 43 & Mandible & Shallow \\
\hline 22 & $\mathrm{~F}$ & 47 & 33 & Mandible & Shallow & 43 & Mandible & Shallow \\
\hline
\end{tabular}

With biomodification and (+) without biomodification (-) with NanoChi

$M$ male, $F$ female

Table 3 Clinical analyzes at baseline ( 7 days) and final ( 1 year) periods after the restorative procedure

\begin{tabular}{|c|c|c|c|c|c|c|c|c|c|c|c|c|c|c|}
\hline \multirow{2}{*}{$\frac{\text { Treatment }}{\text { With } 2.5 \% \text { NanoChi }}$} & \multicolumn{2}{|l|}{ Evaluation period } & \multicolumn{3}{|c|}{ Retention } & \multicolumn{3}{|c|}{ Secondary caries } & \multicolumn{3}{|c|}{ Marginal adaptation } & \multicolumn{3}{|c|}{ Postoperative sensibility } \\
\hline & Baseline (7 days) & $n=22(\%)$ & A & B & $\mathrm{C}$ & A & B & $\mathrm{C}$ & A & B & $\mathrm{C}$ & A & $\mathrm{B}$ & $\mathrm{C}$ \\
\hline & & & 22 & - & - & 22 & - & - & 22 & - & - & 22 & - & - \\
\hline & & & 100 & - & - & 100 & - & - & 100 & - & - & 100 & - & - \\
\hline & Final (1 year) & $n=22(\%)$ & A & B & $\mathrm{C}$ & A & B & $\mathrm{C}$ & A & B & $\mathrm{C}$ & A & B & $\mathrm{C}$ \\
\hline & & & 20 & - & 2 & 22 & - & - & 20 & - & 2 & 22 & - & - \\
\hline & & & 91 & - & 9 & 100 & - & - & 91 & - & 9 & 100 & - & - \\
\hline \multirow[t]{6}{*}{ Without $2.5 \%$ NanoChi } & Baseline (7 days) & $n=22(\%)$ & A & B & $\mathrm{C}$ & A & B & $\mathrm{C}$ & A & B & $\mathrm{C}$ & A & B & $\mathrm{C}$ \\
\hline & & & 22 & - & - & 22 & - & - & 22 & - & - & 22 & - & - \\
\hline & & & 100 & - & - & 100 & - & - & 100 & - & - & 100 & - & - \\
\hline & Final (1 year) & $n=22(\%)$ & A & B & $\mathrm{C}$ & A & B & $\mathrm{C}$ & A & B & $\mathrm{C}$ & A & B & $\mathrm{C}$ \\
\hline & & & 19 & - & 3 & 22 & - & - & 19 & - & 3 & 22 & - & - \\
\hline & & & 86 & - & 14 & 100 & - & - & 86 & - & 14 & 100 & - & - \\
\hline
\end{tabular}

criteria and anatomical form photographic criteria. In the control group, $86 \%$ of the restorations were scored as Alpha and $14 \%$ as Charlie for retention and marginal adaptation clinical criteria and anatomical form photographic criteria. Besides, $100 \%$ of the restorations were scored as Alpha for others clinical (secondary caries and postoperative sensitivity) and photographic (restoration color and marginal pigmentation) criteria in the experimental and control groups (Tables 3 and 4).

Population demographics were computed and Kaplan-Meier survival estimates were calculated for 1 year. The survival of restorations at 1 year is $93 \%$ for teeth 
Table 4 Photographic analyzes at baseline (7 days) and final (1 year) periods after the restorative procedure

\begin{tabular}{|c|c|c|c|c|c|c|c|c|c|c|c|}
\hline \multirow{2}{*}{$\begin{array}{l}\text { Treatment } \\
\text { With } 2.5 \% \text { NanoChi }\end{array}$} & \multicolumn{2}{|l|}{ Evaluation period } & \multicolumn{3}{|c|}{ Restoration color } & \multicolumn{3}{|c|}{ Marginal pigmentation } & \multicolumn{3}{|c|}{ Anatomical form } \\
\hline & Baseline (7 days) & $n=22(\%)$ & $\mathrm{A}$ & $\mathrm{B}$ & $\mathrm{C}$ & A & $\mathrm{B}$ & $\mathrm{C}$ & A & B & $\mathrm{C}$ \\
\hline & & & 22 & - & - & 22 & - & - & 22 & - & - \\
\hline & & & 100 & - & - & 100 & - & - & 100 & - & - \\
\hline & Final (1 year) & $n=22(\%)$ & A & $\mathrm{B}$ & $\mathrm{C}$ & $\mathrm{A}$ & $\mathrm{B}$ & $\mathrm{C}$ & A & $\mathrm{B}$ & $\mathrm{C}$ \\
\hline & & & 22 & - & - & 22 & - & - & 20 & - & 2 \\
\hline & & & 100 & - & - & 100 & - & - & 91 & - & 9 \\
\hline \multirow[t]{6}{*}{ Without $2.5 \%$ NanoChi } & Baseline (7 days) & $n=22(\%)$ & $\mathrm{A}$ & $\mathrm{B}$ & $\mathrm{C}$ & $\mathrm{A}$ & $\mathrm{B}$ & $\mathrm{C}$ & $\mathrm{A}$ & $\mathrm{B}$ & $\mathrm{C}$ \\
\hline & & & 22 & - & - & 22 & - & - & 22 & - & - \\
\hline & & & 100 & - & - & 100 & - & - & 100 & - & - \\
\hline & Final (1 year) & $n=22(\%)$ & A & B & $\mathrm{C}$ & A & B & $\mathrm{C}$ & $\mathrm{A}$ & B & $\mathrm{C}$ \\
\hline & & & 22 & - & - & 22 & - & - & 19 & - & 3 \\
\hline & & & 100 & - & - & 100 & - & - & 86 & - & 14 \\
\hline
\end{tabular}

without biomodification and $95 \%$ for teeth with biomodification. These findings show that survival rates of restorations are high at 1 year after treatment.

At the 1-year interval, no significant difference in survival was noted between groups $(p>0.05)$ with and without eroded dentin biomodification. The success rate with $2.5 \%$ NanoChi was $95.5 \%$, while in the control group (without NanoChi) the success rate was $93.2 \%$.

\section{Discussion}

The use of chitosan products in dentin tissues increases the surface resistance against collagenase degradation [14, 15] and contributes to forming a calcium phosphate layer on demineralized dentin [22]. Chitosan has great anti-erosive potential in enamel and dentin when associated with metal ions and fluoride [23]. The demineralized dentin models have shown that chitosan and calcium phosphate-based nanocomplexes favored the dentin remineralization [24]. At the same time, chitosan-based dentifrices significantly reduced dentin erosion by $24-67 \%$ (extrinsic conditions-citric acid, $\mathrm{pH}$ : 2.5) and 21-40\% (intrinsic conditions- $\mathrm{HCl} / \mathrm{pepsin}$ solution, pH: 1.6) [24, 25].

In enamel, calcium phosphate chitosan nanoparticles positively affect the remineralization process in a similar way to that in the oral cavity, but different from the remineralization that occurs by the fluoride action [26]. In dentin, phosphorylated chitosan increases the deposition of calcium and phosphate ions [22]. However, the chitosan solutions have limitations in reducing dentin surface loss after an erosion challenge compared to fluoride and metallic solutions [27]. The chitosan does not influence the microhardness and $\mathrm{Ca} / \mathrm{P}$ percentage in dentin affected by residual caries [28].
In the present study, the application of the $2.5 \%$ NanoChi did not influence the quality and longevity of the restorations after 1-year based on the USPHS criteria, but the treatment with $2.5 \%$ NanoChi provided a higher percentage of flawless restorations. This result can be justified by only shallow and medium erosion lesions and one tooth surface (palatal or lingual) of patients who were not diagnosed with parafunctional habits or other symptoms resulting from dental erosion. Failures in adhesive restorations may be associated with untreated parafunctional habits that affect occlusions [29], such as bruxism and oral dysfunctions, which may affect oral vertical dimension and limit restorative treatment [30].

The use of the self-etching single bond universal adhesive system may have contributed to the good results obtained of the restorations of erosive lesions since this system chemically bonds to the dentin substrate [31, 32] and is stable in a wet environment [33]. A very important component in this adhesive is the 10-methacryloyloxydecylhydrogen phosphate (10MDP). After self-etch, calcium ions of the hybrid layer upon contact with 10-MDP, chemically interact in nano-layers to form a salt (10-MDP-Ca), which increases the chemical adhesion of the adhesive system to the dental substrate [34] and favors clinical [35, 36] and laboratory [37, 38] results of restorations. As another factor that may have positively affected the results was the enamel-restricted acid etching done before applying the self-etching adhesive system, which increases the bond strength of enamel [39] and dentin [40].

In longer evaluation periods, more obvious failures are observed. Studies have shown that restorative treatments for noncarious cervical lesions, including erosion, present clinical failure after 2-year on average, with a significant deterioration in marginal adaptation and discoloration of the cavosurface margin [41]. Therefore, the fact that biomodification of eroded dentin with $2.5 \%$ NanoChi does not 
influence the clinical behavior of the restorations is favorable because adhesion depends on the adhesive system flow over the surface [31]. Thus, other positive aspects of chitosan as antimicrobial and chelating properties may be present without impairing adherence [42].

Furthermore, recent studies have shown the benefits of using chitosan. For example, chitosan-calcium aluminate support $(\mathrm{CH}-\mathrm{AlCa})$ combined with a dosage of $1 \alpha, 25-$ dihydroxyvitamin D3, provides a bioactive pulp cell microenvironment, which could be a potential tissue engineering system for direct pulp protection [43]. In another study, triclosan-associated chitosan adhesive resin showed greater antibacterial activity immediately and after 6-month, stabilizing the dentin-adhesive interface and maximizing longterm marginal sealing [44]. The chitosan-hydroxyapatite (C-HA) dentin conditioning enhances dentin surface wettability to facilitate tricalcium silicate sealant penetration and dentin tensile strength [45].

Due to the strong indications of the benefits of applying chitosan-based products on dentin, further longitudinal studies should be performed, assessing the long-term impact of this substance on the restorative treatment of eroded teeth.

\section{Conclusions}

The biomodification of eroded dentin with $2.5 \%$ NanoChi did not directly influence the resin restorations based on clinical and photographic criteria after a 1-year follow-up. However, the application of $2.5 \%$ NanoChi on eroded dentin provided a higher percentage of flawless restorations. Even if the difference compared to the untreated group was not significant, further long-term studies are needed to support this outcome.

Acknowledgements The author thanks the São Paulo Research Foundation (FAPESP) for financial support (grants \#2017/25757-8 and \#2017/11582-1).

\section{Compliance with ethical standards}

Conflict of interest The authors declare that they have no conflict of interest.

Ethical approval This study was approved by the Research Ethics Committee of the Ribeirao Preto School of Dentistry, University of São Paulo (61188916.8.0000.5419). All procedures performed in studies involving human participants were in accordance with the ethical standards of the institutional and/or national research committee and with the 1964 Helsinki declaration and its later amendments or comparable ethical standards.

Informed consent Informed consent was obtained from all individual participants included in the study.
Publisher's note Springer Nature remains neutral with regard to jurisdictional claims in published maps and institutional affiliations.

Open Access This article is licensed under a Creative Commons Attribution 4.0 International License, which permits use, sharing, adaptation, distribution and reproduction in any medium or format, as long as you give appropriate credit to the original author(s) and the source, provide a link to the Creative Commons license, and indicate if changes were made. The images or other third party material in this article are included in the article's Creative Commons license, unless indicated otherwise in a credit line to the material. If material is not included in the article's Creative Commons license and your intended use is not permitted by statutory regulation or exceeds the permitted use, you will need to obtain permission directly from the copyright holder. To view a copy of this license, visit http://creativecommons. org/licenses/by/4.0/.

\section{References}

1. Jaeggi T, Lussi A. Prevalence, incidence and distribution of erosion. Monogr Oral Sci. 2014;25:55-73. https://doi.org/10.1159/ 000360973.

2. Shellis RP, Addy M. The interactions between attrition, abrasion and erosion in tooth wear. Monogr Oral Sci. 2014;25:32-45. https://doi.org/10.1159/000359936.

3. Amaechi BT. Dental erosion and its clinical management. 2015. https://doi.org/10.1007/978-3-319-13993-7.

4. Hammoudi W, Trulsson M, Smedberg JI, Svensson P. Phenotypes of patients with extensive tooth wear-a novel approach using cluster analysis. J Dent. 2019;82:22-29. https://doi.org/10.1016/j. jdent.2019.01.001.

5. Tschammler C, Simon A, Brockmann K, Röbl M, Wiegand A. Erosive tooth wear and caries experience in children and adolescents with obesity. J Dent. 2019;83:77-86. https://doi.org/10. 1016/j.jdent.2019.02.005.

6. O'Toole S, Bernabé E, Moazzez R, Bartlett D. Timing of dietary acid intake and erosive tooth wear: a case-control study. J Dent. 2017;56:99-104. https://doi.org/10.1016/j.jdent.2016.11.005.

7. Lussi A, Jaeggi T, Jaeggi-Schärer S. Prediction of the erosive potential of some beverages. Caries Res. 1995;29:349-54. https:// doi.org/10.1159/000262091.

8. Schlueter N, Neutard L, Von Hinckeldey J, Klimek J, Ganss C. Tin and fluoride as anti-erosive agents in enamel and dentine in vitro. Acta Odontol Scand. 2010;68:180-4. https://doi.org/10. 3109/00016350903555395.

9. Ururahy MS, Curylofo-Zotti FA, Galo R, Nogueira LFB, Ramos AP, Corona SAM. Wettability and surface morphology of eroded dentin treated with chitosan. Arch Oral Biol. 2017;75:68-73. https://doi.org/10.1016/j.archoralbio.2016.11.017.

10. Soares DG, Rosseto HL, Basso FG, Scheffel DS, Hebling J, Costa $\mathrm{CA}$, et al. Chitosan-collagen biomembrane embedded with calcium-aluminate enhances dentinogenic potential of pulp cells. Braz Oral Res. 2016;30:e54. https://doi.org/10.1590/18073107BOR-2016.vol30.0054.

11. Tay FR, Pashley DH. Resin bonding to cervical sclerotic dentin: a review. J Dent. 2004;32:173-96. https://doi.org/10.1016/j.jdent. 2003.10.009.

12. Zimmerli B, De Munck J, Lussi A, Lambrechts P, Van Meerbeek B. Long-term bonding to eroded dentin requires superficial bur preparation. Clin Oral Investig. 2012;16:1451-61. https://doi.org/ 10.1007/s00784-011-0650-8.

13. De Souza Costa CA, Hebling J, Scheffel DLS, Soares DGS, Basso FG, Ribeiro APD. Methods to evaluate and strategies to improve the biocompatibility of dental materials and operative techniques. 
Dent Mater. 2014;30:769-84. https://doi.org/10.1016/j.dental. 2014.04.010.

14. Shrestha A, Friedman S, Kishen A. Photodynamically crosslinked and chitosan-incorporated dentin collagen. J Dent Res. 2011;90:1346-51. https://doi.org/10.1177/0022034511421928.

15. Fawzy AS, Nitisusanta LI, Iqbal K, Daood U, Beng LT, Neo J. Chitosan/riboflavin-modified demineralized dentin as a potential substrate for bonding. J Mech Behav Biomed Mater. 2013;17:278-89. https://doi.org/10.1016/j.jmbbm.2012.09.008.

16. Dash M, Chiellini F, Ottenbrite RM, Chiellini E. Chitosan-a versatile semi-synthetic polymer in biomedical applications. Prog Polym Sci. 2011;36:981-1014. https://doi.org/10.1016/j. progpolymsci.2011.02.001.

17. Raafat D, Sahl HG. Chitosan and its antimicrobial potential-a critical literature survey. Micro Biotechnol. 2009;2:186-201. https://doi.org/10.1111/j.1751-7915.2008.00080.x.

18. Şenel S, McClure SJ. Potential applications of chitosan in veterinary medicine. Adv Drug Deliv Rev. 2004;56:1467-80. https:// doi.org/10.1016/j.addr.2004.02.007.

19. Elsaka SE, Elnaghy AM. Antibacterial activity of calcium hydroxide combined with chitosan solutions and the outcomes on the bond strength of RealSeal sealer to radicular dentin. J Biomed Res. 2012;26:193-9. https://doi.org/10.7555/JBR.26.20110136.

20. Rabea EI, Badawy MET, Stevens CV, Smagghe G, Steurbaut W. Chitosan as antimicrobial agent: applications and mode of action. Biomacromolecules. 2003;4:1457-65. https://doi.org/10.1021/ bm034130m.

21. Cvar, J, Ryge G. Criteria for the clinical evaluation of dental restorative materials. USPHS Publication. 1971:790-240.

22. $\mathrm{Xu} \mathrm{Z,} \mathrm{Neoh} \mathrm{KG,} \mathrm{Lin} \mathrm{CC,} \mathrm{Kishen} \mathrm{A.} \mathrm{Biomimetic} \mathrm{deposition} \mathrm{of}$ calcium phosphate minerals on the surface of partially demineralized dentine modified with phosphorylated chitosan. J Biomed Mater Res. 2011;98 B:150-9. https://doi.org/10.1002/jbm.b.31844.

23. Ganss C, Lussi A, Schlueter N. Dental erosion as oral disease. Insights in etiological factors and pathomechanisms, and current strategies for prevention and therapy. Am J Dent. 2012;25:351-64.

24. Chen Z, Cao S, Wang H, Li Y, Kishen A, Deng X, et al. Biomimetic remineralization of demineralized dentine using scaffold of CMC/ACP nanocomplexes in an in vitro tooth model of deep caries. PLoS ONE. 2015;10:1-19. https://doi.org/10.1371/journal. pone. 0116553 .

25. Aykut-Yetkiner A, Attin T, Wiegand A. Prevention of dentine erosion by brushing with anti-erosive toothpastes. J Dent. 2014;42:856-61. https://doi.org/10.1016/j.jdent.2014.03.011.

26. Zhang X, Li Y, Sun X, et al. Biomimetic remineralization of demineralized enamel with nano-complexes of phosphorylated chitosan and amorphous calcium phosphate. J Mater Sci Mater Med. 2014;25:2619-28. https://doi.org/10.1007/s10856-014-52852.

27. Beltrame APCA, Suchyta D, Abd Alraheam I, Mohammed A, Schoenfisch M, Walter R, et al. Effect of phosphorylated chitosan on dentin erosion: an in vitro study. Caries Res. 2018;52:378-86. https://doi.org/10.1159/000486521.

28. Curylofo-Zotti FA, Tanta GS, Zucoloto ML, Souza-Gabriel AE, Corona SAM. Selective removal of carious lesion with Er:YAG laser followed by dentin biomodification with chitosan. Lasers Med Sci. 2017;32:1595-603. https://doi.org/10.1007/s10103-017-2287-6.

29. Lavigne GJ, Khoury S, Abe S, Yamaguchi T, Raphael K. Bruxism physiology and pathology: an overview for clinicians. J Oral Rehabil. 2008;35:476-94. https://doi.org/10.1111/j.1365-2842.2008. 01881.x.
30. Fradeani M1, Barducci G, Bacherini LBM. Esthetic rehabilitation of a severely worn dentition with minimally invasive prosthetic procedures (MIPP). Int J Periodontics Restor Dent. 2012;32:135-47.

31. Da Rosa WLDO, Piva E, Da Silva AF. Bond strength of universal adhesives: a systematic review and meta-analysis. J Dent. 2015;43:765-76. https://doi.org/10.1016/j.jdent.2015.04.003.

32. Perdigão J, Ceballos L, Giráldez I, Baracco B, Fuentes MV. Effect of a hydrophobic bonding resin on the 36-month performance of a universal adhesive - a randomized clinical trial. Clin Oral Investig. 2019. https://doi.org/10.1007/s00784-019-02940-x.

33. Bedran-Russo A, Leme-Kraus AA, Vidal CMP, Teixeira EC. An overview of dental adhesive systems and the dynamic tooth-adhesive interface. Dent Clin North Am. 2017;61:713-31. https://doi.org/10.1016/j.cden.2017.06.001.

34. Mena-serrano A. A new universal simplified adhesive: 6-month clinical evaluation a new universal simplified adhesive: 6-month clinical. Evaluation. 2013;25:0-15. https://doi.org/10.1111/jerd. 12005.

35. Loguercio AD, De Paula EA, Hass V, Luque-Martinez I, Reis A, Perdigão J. A new universal simplified adhesive: 36-month randomized double-blind clinical trial. J Dent. 2015;43:1083-92. https://doi.org/10.1016/j.jdent.2015.07.005.

36. Loguercio AD, Luque-Martinez IV, Fuentes S, Reis A, Muñoz MA. Effect of dentin roughness on the adhesive performance in noncarious cervical lesions: a double-blind randomized clinical trial. J Dent. 2018;69:60-69. https://doi.org/10.1016/j.jdent.2017.09.011.

37. Wagner A, Wendler M, Petschelt A, Belli R, Lohbauer U. Bonding performance of universal adhesives in different etching modes. J Dent. 2014;42:800-7. https://doi.org/10.1016/j.jdent. 2014.04.012.

38. Muñoz MA, Luque-Martinez I, Malaquias P, Hass V, Reis A, Campanha $\mathrm{NH}$, et al. In vitro longevity of bonding properties of universal adhesives to dentin. Oper Dent. 2015;40:282-92. https:// doi.org/10.2341/14-055-L

39. Perdigão J, Dutra-Corrêa M, Saraceni CHC, Ciaramicoli MT, Kiyan VH, Queiroz CS. Randomized clinical trial of four adhesion strategies: 18-month results. Oper Dent. 2012;37:3-11. https://doi. org/10.2341/11-222-C.

40. de Cardoso GC, Nakanishi L, Isolan CP, Jardim PDS, de Moraes RR. Bond stability of universal adhesives applied to dentin using etch-and-rinse or self-etch strategies. Braz Dent J. 2019;30:467-75. https://doi.org/10.1590/0103-6440201902578.

41. Abdalla AIESH. Clinical evaluation of a self-etch adhesive in noncarious cervical lesions. Am J Dent. 2008;21:327-30.

42. Curylofo-Zotti FA, Fernandes MP, Martins AA, Macedo AP, Nogueira LFB, Ramos APCS. Caries removal with Er:YAG laser followed by dentin biomodification with carbodiimide and chitosan: wettability and surface morphology analysis. Microsc Res Technol. 2019. https://doi.org/10.1002/jemt.23395.

43. Bordini EAF, Cassiano FB, Silva ISP, Usberti FR, Anovazzi G, Pacheco LE, et al. Synergistic potential of 1 $\alpha, 25$-dihydroxyvitamin D3 and calcium-aluminate-chitosan scaffolds with dental pulp cells. Clin Oral Investig. 2019. https://doi.org/10. 1007/s00784-019-02906-z.

44. Machado AHS, Garcia IM, Motta AS, Leitune VCB, Collares FM. Triclosan-loaded chitosan as antibacterial agent for adhesive resin. $\mathrm{J}$ Dent. 2019;83:33-39. https://doi.org/10.1016/j.jdent.2019.02.002.

45. Hashmi A, Zhang X, Kishen A. Impact of dentin substrate modification with chitosan-hydroxyapatite precursor nanocomplexes on sealer penetration and tensile strength. J Endod. 2019;45:935-42. https://doi.org/10.1016/j.joen.2019.03.021. 